\title{
A Fast Algorithm for Transistor Folding*
}

\author{
EDWARD Y. C. CHENG ${ }^{\dagger}$ and SARTAJ SAHNI \\ Department of Computer and Information Science and Engineering, University of Florida, Gainesville, FL 32611-6120
}

(Received 13 July 1999; In final form 20 September 1999)

\begin{abstract}
Transistor folding reduces the area of row-based designs that employ transistors of different size. Kim and Kang [1] have developed an $O\left(m^{2} \log m\right)$ algorithm to optimally fold $m$ transistor pairs. In this paper we develop an $O\left(m^{2}\right)$ algorithm for optimal transistor folding. Our experiments indicate that our algorithm runs 3 to 60 times as fast for $m$ values in the range $(100,100,000)$.
\end{abstract}

Keywords and Phrases: Transistor folding; Row-based design; Area minimization; Complexity

\section{INTRODUCTION}

In high-performance circuit design, the transistor sizing problem was investigated widely in the past (for example, [7-10]). The objective of transistor sizing is to reduce the circuit delay by increasing the area of transistors. One by-product of transistor sizing is the generation of layouts of transistors of widely varying size. In row-based layout synthesis ([3-6]), we group $p$ MOS and $n$ MOS transistors together and place them in rows. The layout area for these designs is wasted due to nonuniform cell heights. The layout area required can be reduced by folding large transistors so that their height is reduced. Transistor folding to optimize layout area has been considered in [1] and [2]. Her and Wong [2] have developed an
$O\left(m^{6}\right)$, where $m$ is the number of transistor pairs, dynamic programming algorithm for the general transistor folding problem. (If only $s$ heights are possible for the folded transistors, the complexity of Her and Wong's algorithm is $O\left(\mathrm{~m}^{3} \mathrm{~s}^{3}\right)$. In general, $s$ is $O(m)$.) Kim and Kang [1] have developed a more practical algorithm for the case of row-based designs. The complexity of their algorithm is $O\left(m^{2} \log m\right)$ or $O(s(m+s) \log m)$. They also show that the area of row-based designs can be reduced by as much as $30 \%$ by performing transistor folding. In this paper, we consider the row-based-design transistor-folding problem considered in [1] and develop an $O\left(\mathrm{~m}^{2}\right)$ or $O(s(m+s))$ algorithm to minimize area. We also report on experiments conducted by us that show that our algorithm actually runs much faster than the

\footnotetext{
* This research was supported, in part, by the Army Research Office under grant DAA H04-95-1-0111.

$\dagger$ e-mail: yccheng@ cise.uff.edu

${ }^{\ddagger}$ Corresponding author. e-mail: sahni@cise.ufl.edu
} 
algorithm of [1]. The test circuits used in our experiments have between 100 and 100,000 transistor pairs. So, our tests are similar to those conducted in [1] where the circuits had from 192 to 88,258 transistor pairs.

\section{PROBLEM FORMULATION}

We are given a CMOS circuit with a row of $m$ transistor pairs. Each transistor pair consists of a $p$ MOS transistor and its dual $n$ MOS transistor. Let $p_{i}$ and $n_{i}$, respectively, be the heights of the $p$ MOS and $n$ MOS transistors in the $i$ th pair, $1 \leq i \leq m . p_{i}$ and $n_{i}$ are integers that give transistor height in multiples of the minimum resolution $\lambda$. Figure 1 shows a CMOS circuit with 4 pairs of transistors, $p_{2}=10$ and $n_{2}=12$. If the folding height of $p$ MOS transistors is 4 and that of $n \mathrm{MOS}$ transistors is 3 , then the circuit layout is as in Figure 2. The second $p$ MOS transistor is divided into three columns of height 4,4 and 2 respectively, and the second $n$ MOS transistor is divided into four columns of height 3 each. The area

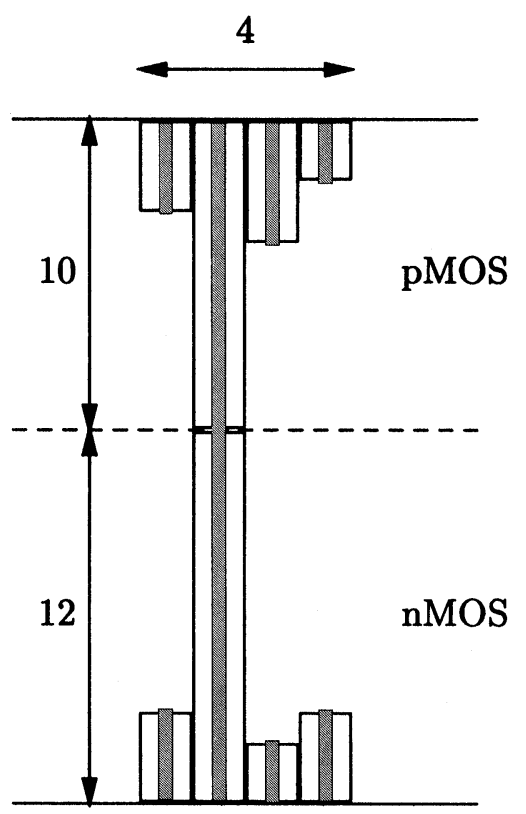

FIGURE 1 An example circuit with 4 pairs of transistors.

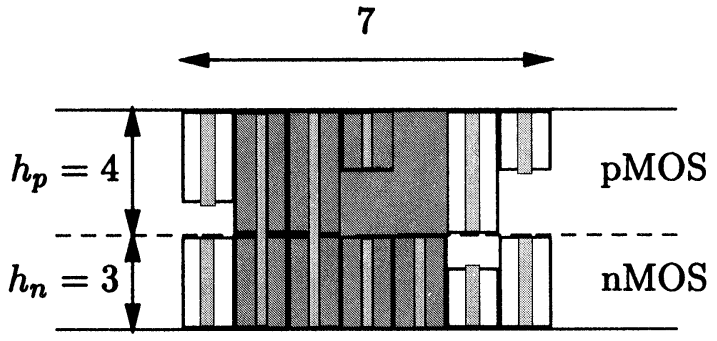

FIGURE 2 The circuit of Figure 1 after folding with $h_{p}=4$ and $h_{n}=3$.

occupied by the folded transistor pair is shown by a shaded box in Figure 2. In practice, the height of the layout area is slightly larger than the sum of the $p$ MOS and $n$ MOS folding heights, and the layout width is slightly larger than the number of transistor columns because of overheads.

Let $h_{p}$ and $h_{n}$ be the folded heights of the $p$ MOS and $n$ MOS transistors, respectively. The height of the folded layout is $h_{p}+h_{n}+c_{v}$ and the width is $\sum_{i=1}^{m} \max \left(\left\lceil\left(p_{i} / h_{p}\right)\right\rceil,\left\lceil\left(n_{i} / h_{h}\right)\right\rceil\right)+c_{h}$ where $c_{v}$ and $c_{h}$ are, respectively, vertical and horizontal overheads. The area of the folded layout is [1]:

$A=\left(h_{p}+h_{n}+c_{v}\right)\left(\sum_{i=1}^{m} \max \left(\left\lceil\frac{p_{i}}{h_{p}}\right\rceil,\left\lceil\frac{n_{i}}{h_{n}}\right\rceil\right)+c_{h}\right)$

In practice, there is a technological constraint on how small $h_{p}$ and $h_{n}$ can be. It is required [1] that $h_{p} \geq P \mathrm{MIN}$ and $h_{n} \geq N \mathrm{MIN}$.

Kim and Kang [1] give two algorithms to determine $h_{p}$ and $h_{n}$ so that the layout area is minimized. The first algorithm is an exhaustive search algorithm that simply tries out all integer choices for $h_{p}$ and $h_{n}$ such that $P \mathrm{MIN} \leq h_{p} \leq \max _{1} \leq i \leq m$ $\left\{p_{i}\right\}=\max (P) \quad$ and $\quad N \mathrm{MIN} \leq h_{n} \leq \max _{1} \leq i \leq m$ $\left\{n_{i}\right\}=\max (N)$. (Here $P=\left\{p_{1}, p_{2}, \ldots, p_{m}\right\}$ and $N=$ $\left\{n_{1}, n_{2}, \ldots, n_{m}\right\}$.) The complexity of the exhaustive search algorithm is $O(\max (P) \cdot \max (N) \cdot m)=$ $O\left(m^{3}\right)$ because $\max (P)$ and $\max (N)$ are $O(m)$ for practical circuits [1].

The second algorithm given in [1] works in two phases. In the first phase, the algorithm constructs a subset $S^{P}$ of $[P \mathrm{MIN}, \max (P)]$ and another subset 
$S^{N}$ of $[N \mathrm{MIN}, \max (N)]$ with the property that the optimal $h_{p}$ is in $S^{P}$ and the optimal $h_{n}$ is in $S^{N}$. The basic observation used to arrive at $S^{P}$ and $S^{N}$ is that if the heights $h_{1}$ and $h_{1}+k$ divide a transistor into the same number of columns then $h_{1}$ is preferred over $h_{1}+k$ (for example if $p_{1}=14$, then folding heights $7,8,9,10,11,12$ and 13 all fold the transistor into two columns; 7 is preferred over the remaining choices). In the second phase the optimal combination $\left(h_{p}, h_{n}\right)$ is determined from $S^{P}$ and $S^{N}$. The complexity of the second phase is $O(s(m+s) \log m)=O\left(m^{2} \log m\right)$, where $s=\left|S^{P}\right|+$ $\left|S^{N}\right|$, and that of the first phase is $\Theta\left(\sum_{i=1}^{m}\left(p_{i}+\right.\right.$ $\left.\left.n_{i}\right)\right)=O\left(m^{2}\right)$ (assuming $\max (P)$ and $\max (N)$ are $O(m))$.

\section{OUR ALGORITHM}

\subsection{Phase I}

Our algorithm is also a two phase algorithm. The first phase of our algorithm is identical to the first phase of Kim and Kang's algorithm [1]. We compute the subsets $S^{P}$ and $S^{N}$ using the code of Figure 3. The arrays $S P L$ and $S N L$ are initialized to zero in the first two for loops. Then we determine the members of $S^{P}$ and $S^{N}$; we set $S P L[i]=1$ if and only if $i \in S^{P}$ and $S N L[i]=1$ if and only if $i \in S^{N}$. Finally, $S^{P}$ and $S^{N}$ are computed in compact form from $S P L$ and $S N L$ respectively. Note that we can compute $S^{P}$ and $S^{N}$ in either ascending or descending order easily by controlling the direction of traversal of the $S N L$ and $S P L$ arrays respectively, in the last two for loops. The algorithm presented in Figure 3 computes $S^{P}$ in ascending order and $S^{N}$ in decending order.

\subsection{Phase II}

Assume that the transistor pairs have been recorded so that $\left(p_{i} / n_{i}\right) \leq\left(\left(p_{i+1}\right) /\left(n_{i+1}\right)\right)$; also assume that $\left(p_{0} / n_{0}\right)=0$ and $\left(\left(p_{m+1}\right) /\left(n_{m+1}\right)\right)=\infty$. The formula, Eq. (1), for the layout area can be rewritten as

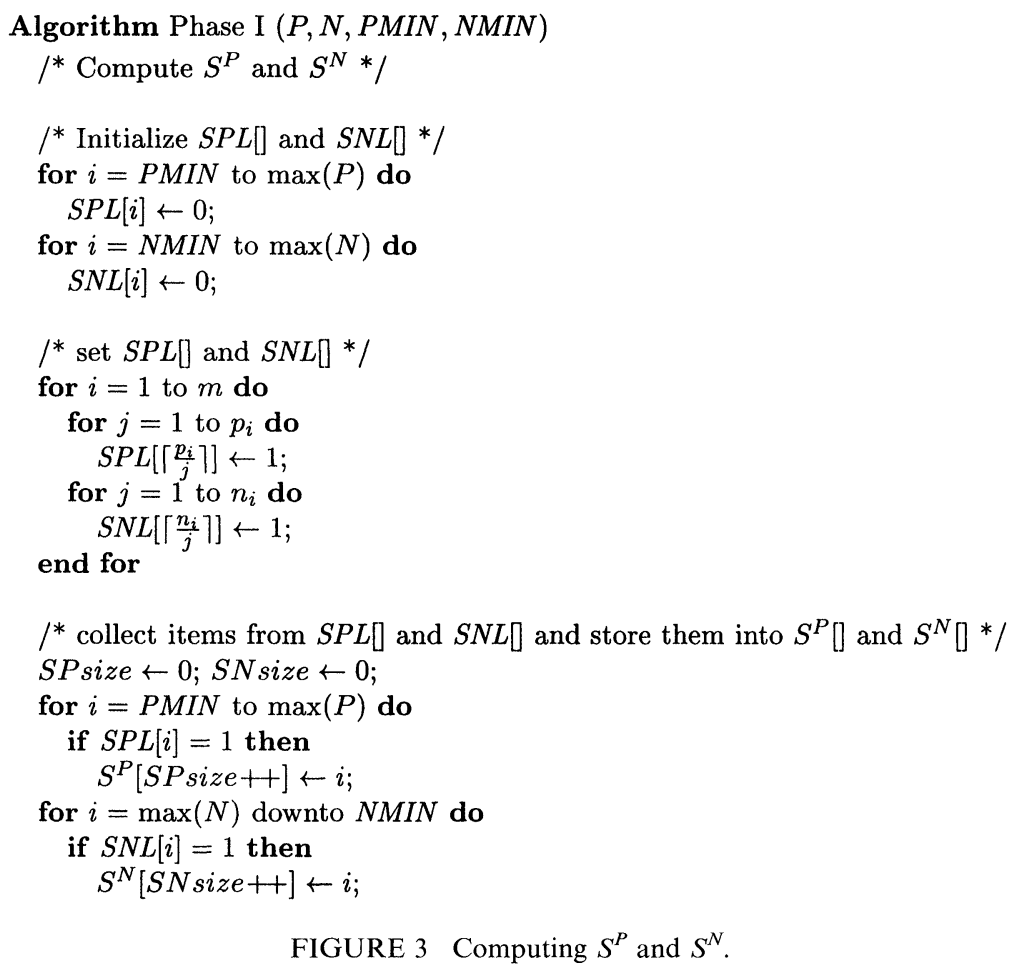




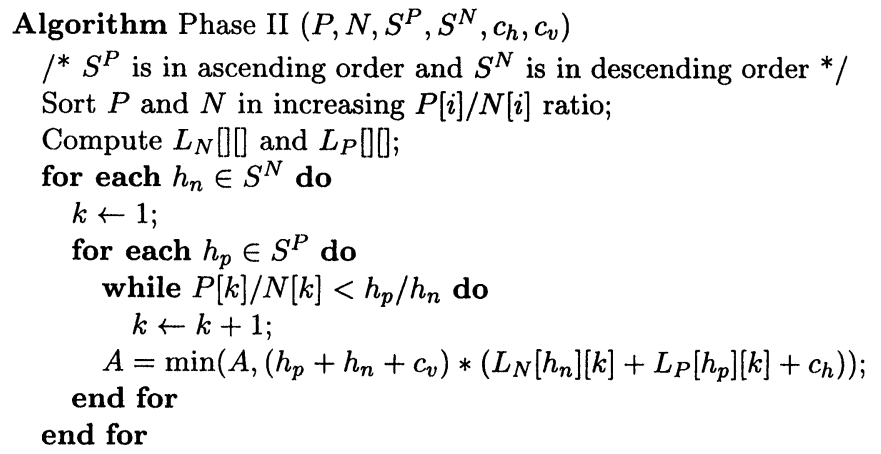

FIGURE 4 Compute optimal $h_{p}$ and $h_{n}$.

$$
A=\left(h_{p}+h_{n}+c_{v}\right)\left(\sum_{i=1}^{k-1}\left\lceil\frac{n_{i}}{h_{n}}\right\rceil+\sum_{i=k}^{m}\left\lceil\frac{p_{i}}{h_{p}}\right\rceil+c_{h}\right)
$$

where $k \in[1, m+1]$ is such that

$$
\frac{p_{k-1}}{n_{k-1}}<\frac{h_{p}}{h_{n}} \leq \frac{p_{k}}{n_{k}}
$$

Let $L_{N}\left(h_{n}, k\right)=\sum_{i=1}^{k-1}\left\lceil\frac{n_{i}}{h_{n}}\right\rceil$ and $L_{P}\left(h_{p}, k\right)=\sum_{i=k}^{m}$ $\left\lceil\left(p_{i} / h_{p}\right)\right\rceil$, we can rewrite Eq. (2) as

$$
A=\left(h_{p}+h_{n}+c_{v}\right)\left(L_{N}\left(h_{n}, k\right)+L_{P}\left(h_{p}, k\right)+c_{h}\right)
$$

From Eq. (3) and the ordering of the transistors by $\left(p_{i} / n_{i}\right)$, it follows that if $h_{n}$ is held fixed and $h_{p}$ increased, the value of $k$ cannot decrease. This observation results in the algorithm of Figure 4.

Since $S^{P}$ and $S^{N}$ can be computed in ascending and descending order respectively by Algorithm Phase I of Figure 3, no sorting is needed to evaluate the members of $S^{P}$ and $S^{N}$ in the specified order. We can sort the transistors into increasing (actually nondecreasing) order of $\left(p_{i} / n_{i}\right)$ in

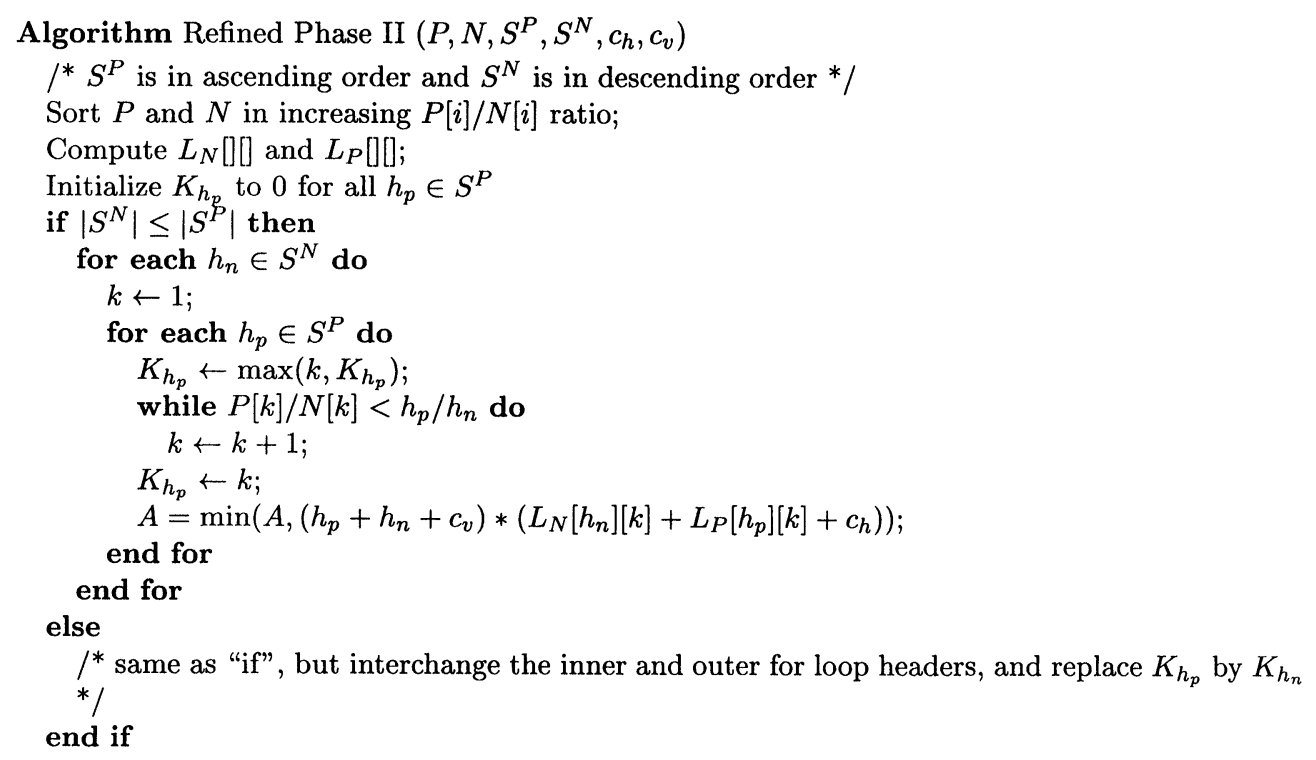

FIGURE 5 Refined Phase 2 algorithm. 
$O(m \log m)$ time; and the arrays $L_{N}$ and $L_{P}$ can be computed in $\Theta\left(m\left|S^{N}\right|\right)$ and $\Theta\left(m\left|S^{P}\right|\right)$ time respectively. Each iteration of the outer for loop takes $O\left(\left|S^{P}\right|+m\right)$ time. Therefore, the time needed for all $\left|S^{N}\right|$ iterations is $O\left(\left|S^{N}\right|\left(\left|S^{P}\right|+m\right)\right)$. We can change this complexity to $O\left(\min \left\{\left|S^{N}\right|,\left|S^{P}\right|\right\} \quad\left(\max \left\{\left|S^{N}\right|\right.\right.\right.$, $\left.\left.\left|S^{P}\right|\right\}+m\right)$ ) by interchanging the inner and outer for loop headers.

Further improvement in run time is possible. Consider the algorithm of Figure 4 . Let $k_{i}$ be the $k$ value that satisfies Eq. (3) when we use the first (i.e., largest) $h_{n}$ value $h_{n_{1}}$ and the $i$ th (i.e., $i$ th smallest) $h_{p}$ value $h_{p_{i}}$. On the next iteration of the outer for loop, $h_{n_{2}} \leq h_{n_{1}}$, so $\left(\left(h_{p_{i}} / h_{n_{1}}\right) \leq\left(h_{p_{t}} / h_{n_{2}}\right)\right)$, and the $k$ value that satisfies Eq. (3) is at least $k_{1}$. Hence if we save $k_{1}$ from the first iteration, we can start the search for the new $k$ value at $k_{1}$. This observation leads to the refinement shown in Figure 5. Although its worstcase complexity is the same as that of Figure 4, it is expected to run faster in practice.

\section{ALGORITHMIC VARIANTS}

Although the use of the preprocessing phase, Phase I, dramatically reduces the run time when processing is completed using the technique of Kim and Kang [1], it is possible that the cost of this preprocessing when used in conjunction with our refined Phase II algorithm of Figure 5 may exceed the benefits that accrue from the preprocessing. Therefore, we formulate another version of our algorithm which does not employ the Phase I preprocessing algorithm of Figure 3. The new version, Figure 6 is just the algorithm of Figure 5 with the while loops replaced by for loops.

Figure 7 shows a variant of the algorithm of Figure 6 in which only one of the arrays $L_{P}$ and $L_{N}$ is precomputed, the values of the other array are computed as needed. This variant reduces the space requirements of the algorithm, and, as we shall see, also reduces the time requirements.

\section{EXPERIMENTAL RESULTS}

The phase 1 algorithm of Figure 3, the phase 2 algorithm of Figure 5, the algorithmic variants of Figures 6 and 7, and the two algorithms of [1] were implemented, by us, in $\mathrm{C}$ and run on a SUN SPARCstation 4. Similar programming

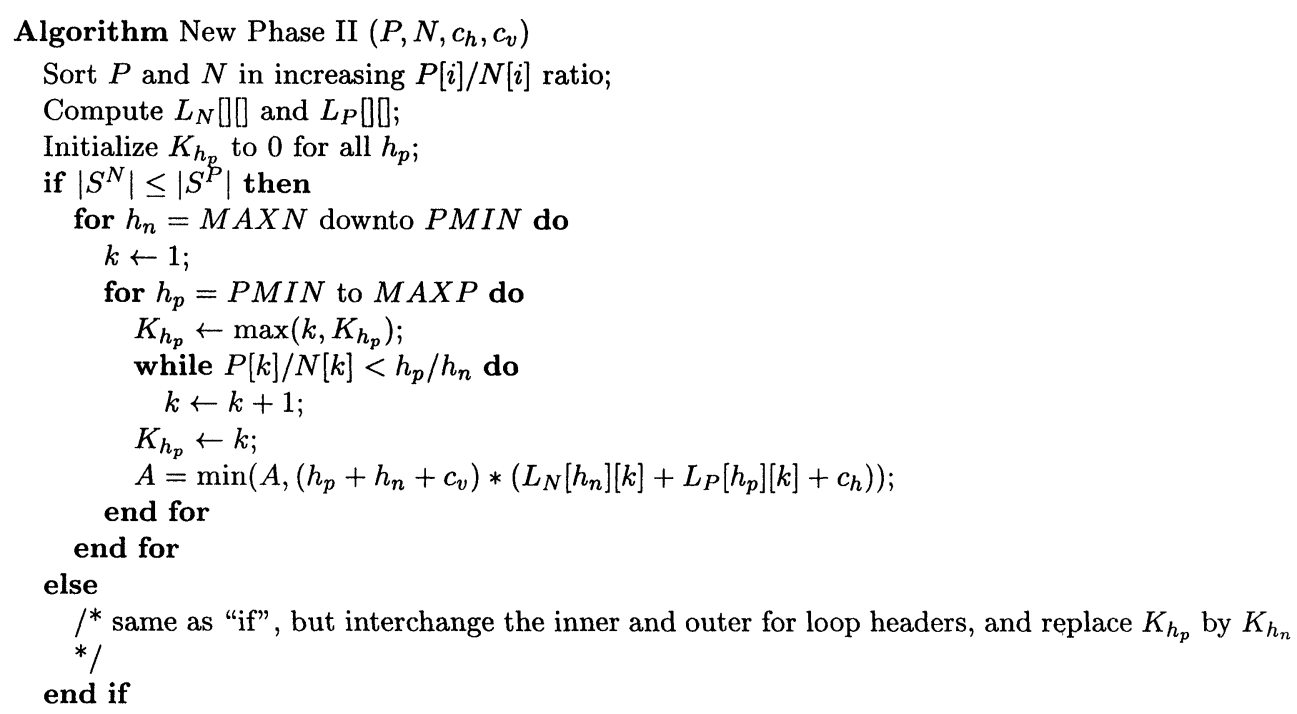

FIGURE 6 New algorithm without Phase I. 


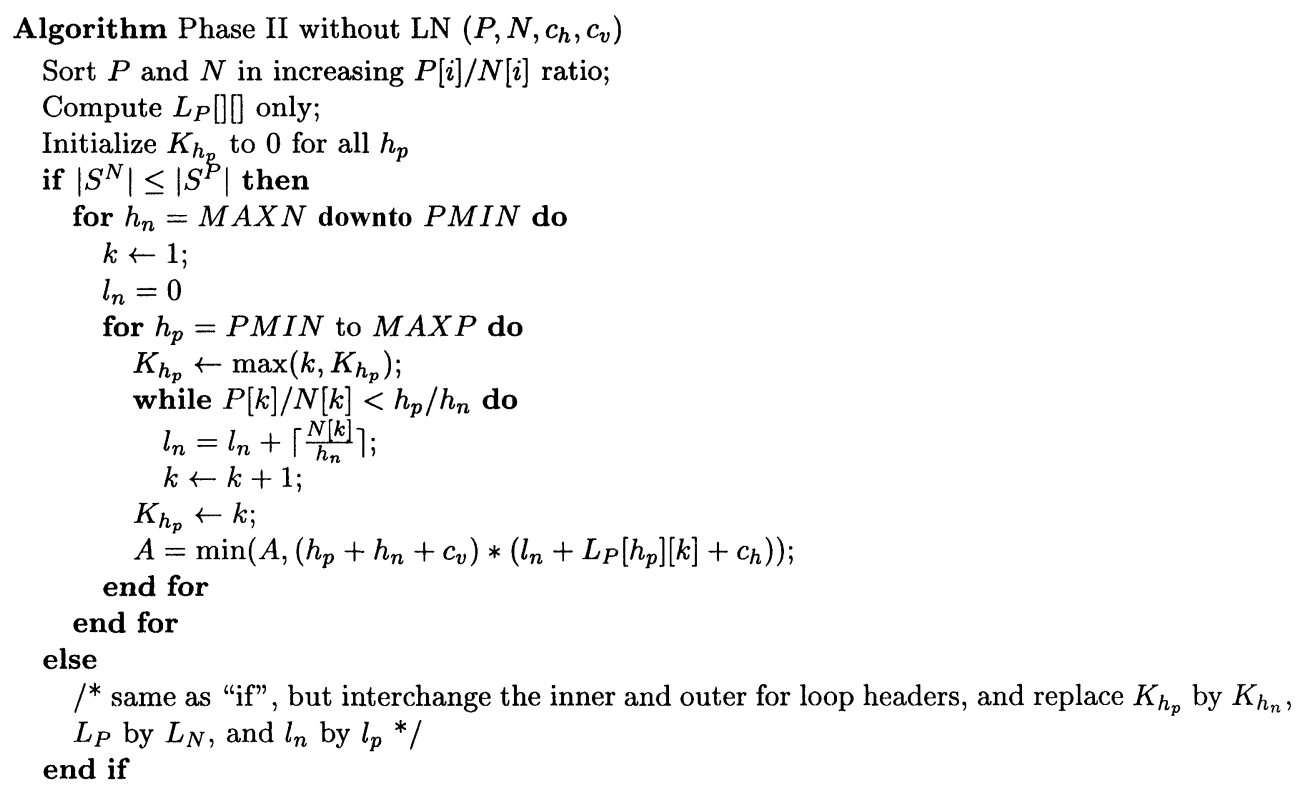

FIGURE 7 New algorithm without Phase I and LN[] [] precomputation.

methodologies were used to develop the codes for our algorithms and those of [1]. As a result, we expect that almost all of the performance difference exhibited in our experiments is due to algorithmic rather than programming differences. Since we were unable to obtain the test data used in [1], we generated random data. We ignore any possible correlation between $p$ MOS and $n$ MOS transistors. For our test data, the number of transistor pairs ranged from 100 to 100,000 . This covers the range in transistor numbers $(192$ to 88,258$)$ in the circuits of [1]. For our first test set, the sizes of the $p$ MOS and $n$ MOS transistors were generated using a uniform random number generator with range $[30,90]$ for $p$ MOS and $[20,60]$ for $n$ MOS. These size ranges correspond to those for the circuit fract that was used in [1], the circuit fract has 598 transistors. Since all of the tested algorithms generate optimal solutions, run time is the only comparative factor. This time is provided in Table $I$. Other than in the column labeled "Phase 1", all times are the times needed for the entire area minimization process (i.e., phase 1 plus phase 2). The exhaustive search algorithm was not run for $m>10,000$ as its run time becomes prohibitive.
In the case of the algorithm proposed in [1], the phase 2 time is significantly larger than the phase 1 time. Our algorithm for phase 2 (5) has brought this time down to approximate the phase 1 time. Equally interestingly, on the data sets used by us, the preprocessing of phase 1 is no longer of any use. When this preprocessing is eliminated (as in Fig. 6), the run time reduces by an additional $30 \%$. The variant of Figure 7 provides modest run time improvement (due mainly to not having to reference a two-dimensional array to get one of the $L$ values), but provides a $50 \%$ reduction in space needs.

For small circuits ( $m \leq 10,000)$, our algorithm of Figure 7 provides a speedup of 3.8 to 7.1 over the algorithm of [1]. On larger circuits, the speedup is more dramatic. For instance, when $m=100,000$ our algorithm of Figure 7 is almost 27 times as fast as that of [1].

We experimented with two other data sets. Table II reports the run times for circuits in which the range of the uniform random number generator was set to $[30,180]$ for $p$ MOS transistor sizes and $[20,120]$ for $n$ MOS sizes and Table III gives the run times when the transistor sizes are 
TABLE I Run time using a uniform distribution

\begin{tabular}{lcccccc}
\hline \multicolumn{7}{c}{ Time in seconds } \\
\cline { 2 - 7 }$m$ & Exhaustive & Phase 1 & Kim and Kang & Figure 5 & Figure 6 & Figure 7 \\
\hline 100 & 1.46 & 0.02 & 0.50 & 0.09 & 0.07 & 0.07 \\
300 & 4.41 & 0.07 & 0.92 & 0.27 & 0.19 & 0.19 \\
500 & 7.34 & 0.11 & 1.29 & 0.44 & 0.32 & 0.30 \\
600 & 8.79 & 0.15 & 1.48 & 0.53 & 0.38 & 0.37 \\
1000 & 14.67 & 0.25 & 2.35 & 0.88 & 0.63 & 0.61 \\
5000 & 74.59 & 1.20 & 13.54 & 4.48 & 3.17 & 3.03 \\
10000 & 149.12 & 2.43 & 35.09 & 9.22 & 6.43 & 6.22 \\
50000 & - & 12.42 & 473.72 & 47.94 & 32.12 & 30.31 \\
100000 & - & 24.24 & 1710.36 & 96.33 & 69.48 & 63.58 \\
\hline
\end{tabular}

TABLE II Run time using a uniform distribution with larger limits

\begin{tabular}{lcccccc}
\hline \multicolumn{7}{c}{ Time in seconds } \\
\cline { 2 - 6 }$m$ & Exhaustive & Phase 1 & Kim and Kang & Figure 5 & Figure 6 & Figure 7 \\
\hline 100 & 6.35 & 0.05 & 1.73 & 0.16 & 0.17 & 0.16 \\
300 & 19.87 & 0.13 & 3.36 & 0.54 & 0.43 & 0.42 \\
500 & 33.15 & 0.22 & 4.44 & 0.91 & 0.69 & 0.66 \\
600 & 39.77 & 0.26 & 4.95 & 1.09 & 0.83 & 0.80 \\
1000 & 66.31 & 0.44 & 6.67 & 1.81 & 1.36 & 1.29 \\
5000 & 336.92 & 2.19 & 26.71 & 9.24 & 6.74 & 6.41 \\
10000 & 673.43 & 4.44 & 57.63 & 18.64 & 13.42 & 12.72 \\
50000 & - & 22.05 & 516.08 & 98.22 & 75.97 & 65.80 \\
100000 & - & 44.17 & 3777.96 & 208.33 & 158.13 & 139.05 \\
\hline
\end{tabular}

TABLE III Run time using a normal distribution

\begin{tabular}{lcccccc}
\hline \multicolumn{7}{c}{ Time in seconds } \\
\cline { 2 - 7 }$m$ & Exhaustive & Phase 1 & Kim and Kang & Figure 5 & Figure 6 & Figure 7 \\
\hline 100 & 0.90 & 0.02 & 0.22 & 0.04 & 0.03 & 0.03 \\
300 & 3.22 & 0.06 & 0.53 & 0.13 & 0.10 & 0.09 \\
500 & 5.79 & 0.10 & 0.83 & 0.21 & 0.17 & 0.16 \\
600 & 6.70 & 0.12 & 1.00 & 0.25 & 0.20 & 0.19 \\
1000 & 12.69 & 0.19 & 1.68 & 0.43 & 0.36 & 0.34 \\
5000 & 68.26 & 0.96 & 13.67 & 2.42 & 1.89 & 1.77 \\
10000 & 129.67 & 1.92 & 38.07 & 4.79 & 3.65 & 3.41 \\
50000 & - & 9.60 & 677.42 & 27.20 & 19.29 & 18.04 \\
100000 & - & 19.22 & 2663.71 & 59.49 & 45.33 & 40.12 \\
\hline
\end{tabular}

from a normal distribution with mean 40 and standard deviation 10 for $p$ MOS transistors and mean 30 and standard deviation 10 for $n \mathrm{MOS}$ transistors. The speedups range from a low of 4.1 to a high of 66.3 .

\section{CONCLUSION}

We have developed a transistor folding algorithm that is both theoretically and practically faster than the algorithm proposed in [1]. Our 
algorithm is also simpler to code. Experiments suggest that our algorithm runs 3 to 60 times as fast as the algorithm of [1] on circuits with 100 to 100,000 transistor pairs. These circuit sizes are comparable to those used in [1].

\section{References}

[1] Jaewon Kim and Kang, S. M. (1997). An efficient transistor folding algorithm for row-based CMOS layout design. In: Proceedings 34th Design Automation Conference, pp. 456-459.

[2] Her, T. W. and Wong, D. F., Cell area minimization by transistor folding. In: Proceedings 1993 Euro-DAC, pp. $172-177$.

[3] Hsieh, Y. C., Hwang, C. Y., Lin, Y. L. and Hsu, Y. C. (1991). LiB: A CMOS cell compiler, IEEE Transactions on Computer-Aided Design, 10(8).

[4] Stauffer, A. and Nair, R., Optimal CMOS cell transistor placement: A relaxation approach. In: IEEE International Conference on Computer-Aided Design, November, 1988.

[5] Uehara, T. and VanCleemput, W. (1981). Optimal layout of CMOS functional arrays, IEEE Transactions on Computers, C-30(5).

[6] Wimer, S., Pinter, R. Y. and Feldman, J. A. (1987). Optimal chaining of CMOS transistors in a functional cell, IEEE Transactions on Computer-Aided Design, 30(5).

[7] Sachin S. Sapatnekar, Vasant B. Rao, Pravin M. Vaidya and Sung-Mo Kang., An exact solution to the transistor sizing problem for CMOS circuits using convex optimization, IEEE Transaction on Computer-Aided Design, 12(11), 1621-1634, November, 1993.

[8] Fishburn, J. P. and Dunlop, A. E., TILOS: A posynomial programming approach to transistor sizing. In: IEEE International Conference on Computer-Aided Design, pp. 326-328, November, 1985.

[9] Shyu, J., Fishburn, J. P., Dunlop, A. E. and SangiovanniVincentelli, A. L., Optimization-based transistor sizing. IEEE Journal on Solid-State Circuits, pp. 400-409, April, 1988.

[10] Dai, Z. and Asada, K., Mosiz: A two-step transistor sizing algorithm based on optimal timing assignment method for multi-stage complex gates. In: Proceedings 1989 Custom Integrated Circuits Conference, pp. 17.3.1-17.3.4, May, 1989.

\section{Authors' Biographies}

Sartaj Sahni is a Distinguished Professor of Computer and Information Sciences and Engineering at the University of Florida. He is also a Fellow of IEEE, ACM, AAAS, and Minnesota Supercomputer Institute. Dr. Sahni received his B.Tech. (Electrical Engineering) degree from the
Indian Institute of Technology, Kanpur, and the M.S. and Ph.D. degrees in Computer Science from Cornell University. Dr. Sahni has published over one hundred and fifty research papers and written several texts. His research publications are on the design and analysis of efficient algorithms, parallel computing, interconnection networks, and design automation.

Dr. Sahni is a coauthor of the texts: Fundamentals of Data Structures, Fundamentals of Data Structures in Pascal, Fundamentals of Data Structures in C, Fundamentals of Data Structures in $\mathrm{C}++$, Fundamentals of Computer Algorithms, and Hypercube Algorithms: With Applications to Image Processing and Pattern Recognition and author of the texts: Concepts in Discrete Mathematics, Software Development in Pascal, and Data Structures, Algorithms, and Applications in C ++ . In 1997, he was awarded the IEEE Taylor L. Booth Education Award "for contributions to Computer Science and Engineering education in the areas of data structures, algorithms, and parallel algorithms".

Dr. Sahni is a co-editor of the Journal of Parallel and Distributed Computing and is on the editorial boards of IEEE Parallel and Distributed Technology, and Computer Systems: Science and Engineering. He has served as program committee chair, general chair, and been a keynote speaker at many conferences.

Edward Cheng received the Bachelor of Engineering degree in computer engineering from the University of Hong Kong, Hong Kong, in 1992, the Master of Philosophy degree in computer science from the Hong Kong University of Science and Technology, Hong Kong, in 1994, and the Doctor of Philosophy degree from the Department of Computer and Information Science and Engineering, the University of Florida, Gainesville, Florida, in 1998. He is currently with Actel Corporation at Sunnyvale. California, His research interest include VLSI CAD, algorithm design and theory of computation. 

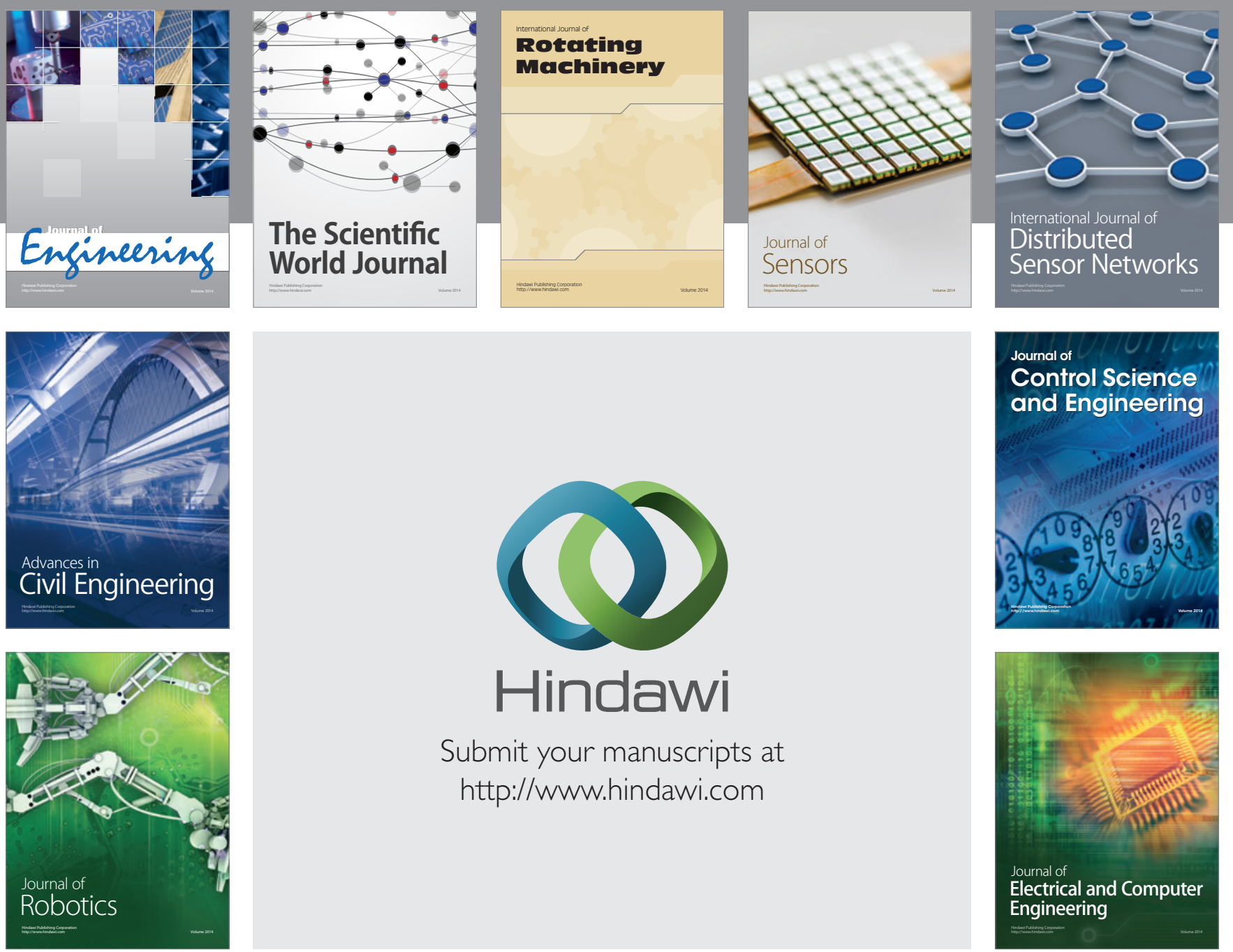

Submit your manuscripts at

http://www.hindawi.com
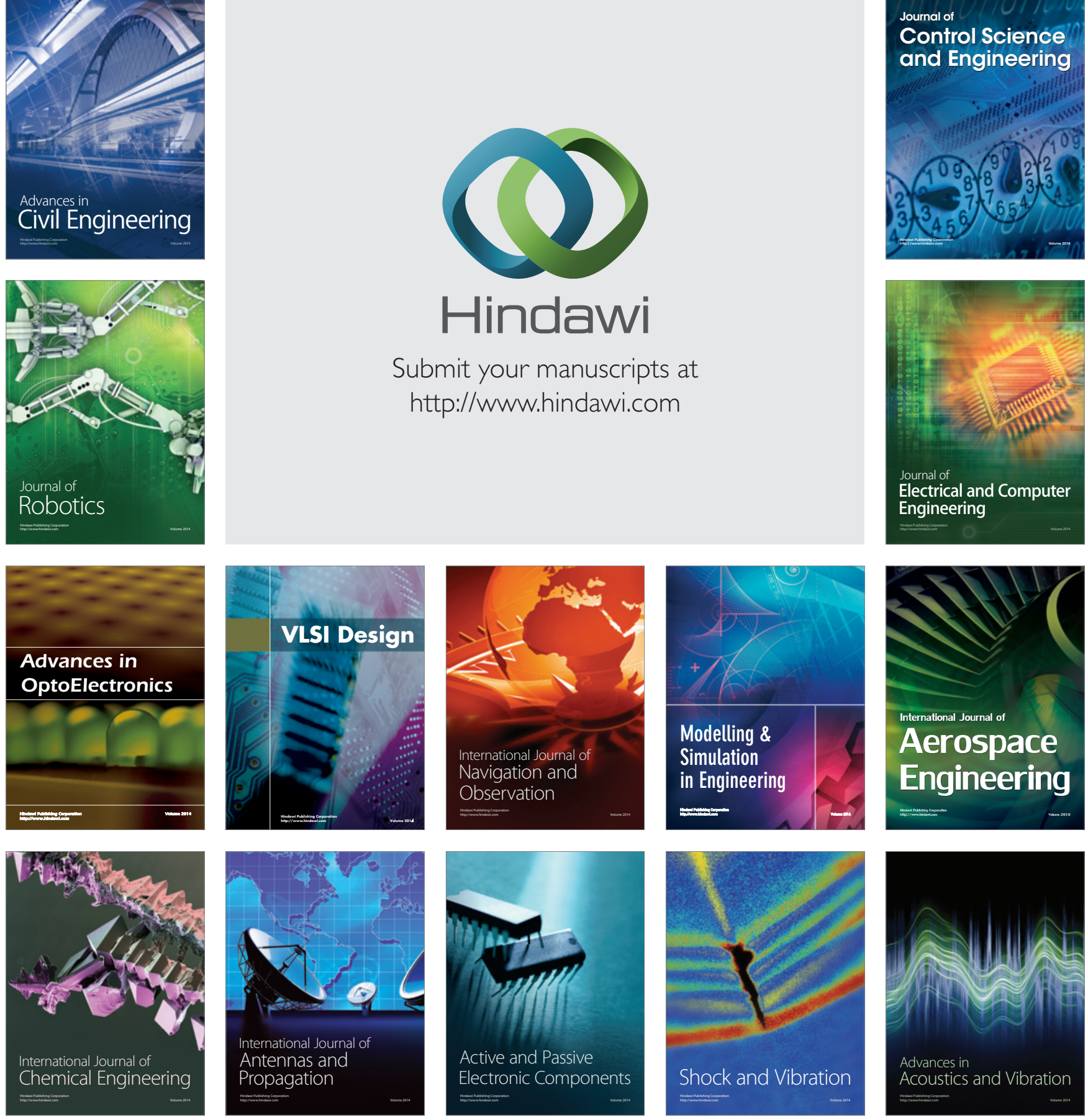